\title{
Dynamic Analysis of a Selected Spatial One-DOF Linkage Mechanism with Friction in Joints
}

\author{
Andrzej Harlecki and Andrzej Urbaś \\ Department of Mechanics, Faculty of Mechanical Engineering and Computer Science, University of Bielsko-Biala, Willowa 2,
} Bielsko-Biala 43-309, Poland

\begin{abstract}
The dynamic analysis of a one-DOF RSRRR spatial linkage mechanism, including four rotational joints R and one spherical joint $S$, is presented in the paper. It is assumed that friction occurs in the rotational joints, whereas a spherical joint can be treated as an ideal one. The mechanism in the form of a closed-loop kinematic chain was divided by cut joint technique into two open-loop kinematic chains in place of the spherical joint. Joint coordinates and homogeneous transformation matrices were used to describe the geometry of the system. Equations of the chains' motion were derived using formalism of Lagrange equations. Cut joint constraints and reaction forces, acting in the cutting place-i.e. in the spherical joint, have been introduced to complete the equations of motion. As a consequence, a set of differential-algebraic equations has been obtained. In order to solve these equations, a procedure based on differentiation twice of the formulated constraint equations with respect to time has been applied. In order to determine values of friction torques in the rotational joints in each integrating step of the equations of motion, joint forces and torques were calculated using the recursive Newton-Euler algorithm taken from robotics. For the requirements of the method, a model of a rotational joint has been developed. Some examples of results of the numerical calculations made have been presented in the conclusions of the paper.
\end{abstract}

Key words: Dynamic analysis, linkage mechanism, friction, cut joint technique.

\section{Nomenclature}

$c$
$g$
$p$
$a^{(c, p)}$
$\bar{a}^{(c, p)}$
$d^{(c, p)}$
$\left.d_{\alpha}^{(c, p)}\right|_{\alpha \in\{A, B, C\}}$
$k^{(c, p)}$
$l^{(c, p)}$
$n^{(c, p)}$

Kinematic chain index

Acceleration of gravity

Link index

Length of journal (bearing liner) of rotational joint connecting link $p$ with link $p-1$

Spacing of friction rotational surfaces of journal (bearing liner) of rotational joint connecting link $p$ with link $p-1$

Link diameter

Respectively diameters of rotational friction surfaces $A, B, C$ of rotational joint connecting link $p$ with link $p-1$

Coefficient defining form of kinetic friction characteristic in rotational joint connecting link $p$ with link $p-1$

Link length

Link mass

Number of links in kinematic chain $c$

Corresponding author: Andrzej Harlecki, professor, research field: dynamics of machines.

$t_{d r, 0}^{(1,1)}$
$t_{s t}$
$\tilde{\mathbf{f}}_{C^{(c, p)}}^{(c, p)}$
$\tilde{\mathbf{f}}_{O^{(c, p)}}^{(c, p)}$
$\tilde{\mathbf{n}}_{C^{(c, p)}}^{(c, p)}$
$\tilde{\mathbf{n}}_{O^{(c, p)}}^{(c, p)}$
$\tilde{\mathbf{r}}_{A}^{(c, p)}$
$\mathbf{r}_{A}^{(c, p)}$
$(\mathbf{r})_{\alpha}$
$\mathbf{t}_{d r}^{(1,1)}$
$\left.\mathbf{t}_{f, \alpha}^{(c, p)}\right|_{\alpha \in\{A, B, C\}}$
$\mathbf{t}_{r e s}^{(1,1)}$
$\mathbf{H}^{(c, p)}$

$\mathbf{H}^{(c, p)}$
Parameter defining time course of value of driving torque (it determines the value of this torque after starting time)

Starting time

Force with opposite sense to inertial force of link $p$

Joint forces exerted on link $p$ by link $p-1$

Torque with opposite sense to inertial torque of link $p$ at centre of mass $C^{(c, p)}$

Joint torque exerted on link $p$ by link $p-1$

Vector of position of point $A$ in local coordinate system

Vector of position of point $A$ in global reference system $\{1,0\}$

Component $\alpha$ of vector $\mathbf{r}$

Driving torque

Kinetic friction torque respectively on rotational friction surfaces $A, B, C$ of bearing liner of rotational joint connecting link $p$ with link $p-1$

Resistance torque

Link pseudo-inertial matrix $4 \times 4$ 


\begin{tabular}{|c|c|}
\hline $\mathbf{I}^{(c, p)}$ & Link inertial matrix $3 \times 3$ \\
\hline$\tilde{\mathbf{R}}^{(c, p)}$ & $\begin{array}{l}\text { Rotation matrix } 3 \times 3 \text { from local } \\
\text { coordinate system of link } p \text { to system } \\
\text { of link } p-1\end{array}$ \\
\hline$\tilde{\mathbf{T}}^{(c, p)}$ & $\begin{array}{l}\text { Transformation matrix } 4 \times 4 \text { from } \\
\text { local coordinate system of link } p \text { to } \\
\text { system of link } p-1\end{array}$ \\
\hline $\mathbf{T}^{(c, p)}$ & $\begin{array}{l}\text { Transformation matrix } 4 \times 4 \text { from } \\
\text { local coordinate system of link } p \text { to } \\
\text { global reference system }\{1,0\}\end{array}$ \\
\hline$(\mathbf{T})_{\alpha, \beta}$ & $\begin{array}{l}\text { Element of matrix } \mathbf{T} \text { being on } \\
\text { intersection of row } \alpha \text { and column } \beta \\
\mathbf{T}_{i}^{(c, p)}=\frac{\partial \mathbf{T}^{(c, p)}}{\partial q_{i}}, \quad \mathbf{T}_{i, j}^{(c, p)}=\frac{\partial^{2} \mathbf{T}^{(c, p)}}{\partial q_{i} \partial q_{j}}\end{array}$ \\
\hline$\dot{\psi}_{0}^{(c, p)}$ & $\begin{array}{l}\text { Parameter defining time course of value } \\
\text { of resistance torque } \\
\text { Coefficient defining form of kinetic } \\
\text { friction characteristic in rotational joint } \\
\text { connecting link } p \text { with link } p-1 \text { (it }\end{array}$ \\
\hline$\dot{\psi}_{k}^{(c, p)}$ & $\begin{array}{l}\text { determines the value of relative velocity } \\
\text { in this joint when kinetic friction } \\
\text { coefficient } \mu_{\alpha}^{(c, p)} \text { assumes a practically } \\
\text { constant value) }\end{array}$ \\
\hline & $\begin{array}{l}\text { Coefficient defining form of kinetic } \\
\text { friction characteristic in rotational joint }\end{array}$ \\
\hline$\mu_{\alpha, 0}^{(c, p)}$ & $\begin{array}{l}\text { connecting link } p \text { with link } p-1 \text { (it } \\
\text { determines the maximal value of kinetic } \\
\text { friction coefficient) }\end{array}$ \\
\hline$\left.\mu_{\alpha}^{(c, p)}\right|_{\alpha \in\{A, B, C\}}$ & $\begin{array}{l}\text { Kinetic friction coefficient on rotational } \\
\text { friction surfaces of pin (bearing liner) } \\
\text { connecting } p \text { link with } p-1 \text { link }\end{array}$ \\
\hline$\tilde{\boldsymbol{\omega}}^{(c, p)}$ & $\begin{array}{l}\text { Angular velocity of link } p \text { defined in } \\
\text { local coordinate system }\end{array}$ \\
\hline$\dot{\tilde{\boldsymbol{\omega}}}^{(c, p)}$ & $\begin{array}{l}\text { Angular acceleration of link } p \\
\text { defined in local coordinate system }\end{array}$ \\
\hline$\dot{\tilde{\mathbf{v}}}_{C^{(c, p)}}^{(c, p)}$ & $\begin{array}{l}\text { Acceleration of centre of mass } C^{(c, p)} \\
\text { of link } p \text { defined in local coordinate } \\
\text { system }\end{array}$ \\
\hline$\dot{\tilde{\mathbf{v}}}_{O^{(c, p)}}^{(c, p)}$ & $\begin{array}{l}\text { Acceleration of origin of local } \\
\text { coordinate system of link } p \text { defined in } \\
\text { this system }\end{array}$ \\
\hline DOF & Degree(s) of freedom \\
\hline
\end{tabular}

\section{Introduction}

The Uicker's doctoral dissertation was the first frequently quoted publication dealing with the dynamic analysis of the spatial linkage mechanism, being the closed-loop kinematic chains [1]. In the proposed method, the equations of motion were formulated on the basis of the Lagrange formalism. The author used here, for the first time, homogeneous transformation matrices which were determined according to an approach presented by Denavit and Hartenberg [2]. As one of the examples, he proposed a dynamic analysis of a two-DOF RSSR classical spatial mechanism containing two one-DOF rotational joints $\mathrm{R}$ and two three-DOF spherical joints $\mathrm{S}$. The dynamics of this kind of the mechanism-popular in the technical applications - was also analyzed in later years, among others, by the authors of the publications $[3,4]$.

Since that time, the dynamics of spatial linkage mechanisms has been analyzed in many works. As it is stated in publication [5], there are several different approaches to the dynamic analysis of such mechanisms. One of the main criteria enabling to classify them is a way of taking into account the coordinates determining the mechanism geometry. Many authors use "relative" coordinates. These determine the position of a link of the mechanism with respect to the previous one in the kinematic chain. Other authors use "reference point" coordinates. In this approach the position of a link is determined by the Cartesian coordinates of a point and by Euler angles or by Euler parameters (called also "quaternions") that define angular orientation of the link. The authors of the work [6] described a new system of coordinates called "natural coordinates" that, in their opinion, have some important advantages in comparison to the "relative" and "reference point" coordinates.

The subject of the analysis presented in this work is a one-DOF RSRRR spatial linkage mechanism. It has one spherical joint $S$ and four rotational joints $R$. In the work [7], a method of the dynamic analysis of this type of the mechanism is presented, but other formalism was used, that is the d'Alembert principle. In the article [8], a dynamic analysis method of one-DOF PUSR spatial linkage mechanism (where P means a one-DOF prismatic joint and $\mathrm{U}-\mathrm{a}$ two-DOF universal joint, in fact being a composition of two rotational joints R), presented in the work [9], has been analyzed. The authors based here on the assumptions included in the works [10-12]. Both 
characterized mechanisms, in fact being a family of the spatial linkage mechanisms, contain neither redundant DOF nor passive constraints. They can have a one three-DOF spherical joint and another one-DOF joint.

\section{A Description of the Dynamic Analysis Methods}

In the accepted method, friction is taken into account in the all rotational joints $\mathrm{R}$ of the mechanism, whereas the spherical joint $\mathrm{S}$ is treated as an ideal one. It is assumed that the mechanism is cut in the spherical joint obtaining two open kinematic chains joined with the fixed base. In the cutting place applicable reaction forces acting on the formed chains are introduced. The Denavit-Hartenberg notation is used to describe the geometry of these chains. This notation bases on homogeneous transformation matrices $4 \times 4$ and joint coordinates (defining relative position of links) according to the methodology presented in the work [13] which deals with a description of the geometry of robot manipulators. Since it can be seen easily that the structure of the obtained chains is analogical to the structure of the manipulators, which are modelled in the form of open-loop kinematic chains, thus the procedure regarding formulating equations of the chains' motion can be analogical to the approach which is used in the case of the dynamic analysis of the manipulators. In the accepted method, the equations of the motion of chains are determined by using formalism of the Lagrange equations on the basis of algorithms provided in the monograph [14]. In order to determine unknown reaction forces in cutting place of the mechanism, additional equations of geometrical constraints are formulated. These equations (in the form of algebraic equations of second order) are joined to the equations of motion (in the form of ordinary differential equations of second order) to determine unknown values of these forces. A system of the differential-algebraic equations is formed in such a way. In order to determine the values of joint forces and torques, and then the values of kinetic friction torques, in each integrating step of the equations of motion, additional calculations are performed using the recursive Newton-Euler algorithm presented in the monograph [13]. In practice, solving the differential-algebraic equations is relatively complex and usually, by differentiation twice with respect to time of the algebraic equations of the constraints, a final system of ordinary differential equations is obtained. These equations can be solved by using any numerical methods with a relatively large integration step. If it turns out to be necessary, a method of constraint stabilization can be used to improve accuracy of the results of the numerical calculations [15].

\section{An Example of the Analysis}

As mentioned previously, the described method was used to analyze, taken from the work [7], a spatial RSRRR linkage mechanism built of four movable links joined with a fixed base. The number of DOF of the mechanism is: $4 \cdot 6-4 \cdot 5-3=1$. The driving link of the mechanism is loaded by the driving torque $\mathbf{t}_{d r}^{(1,1)}$ and the resistance torque $\mathbf{t}_{\text {res }}^{(1,1)}$ (Fig. 1).

As it is shown in Fig. 2, the mechanism considered was divided in the place of the spherical joint $S$, and it

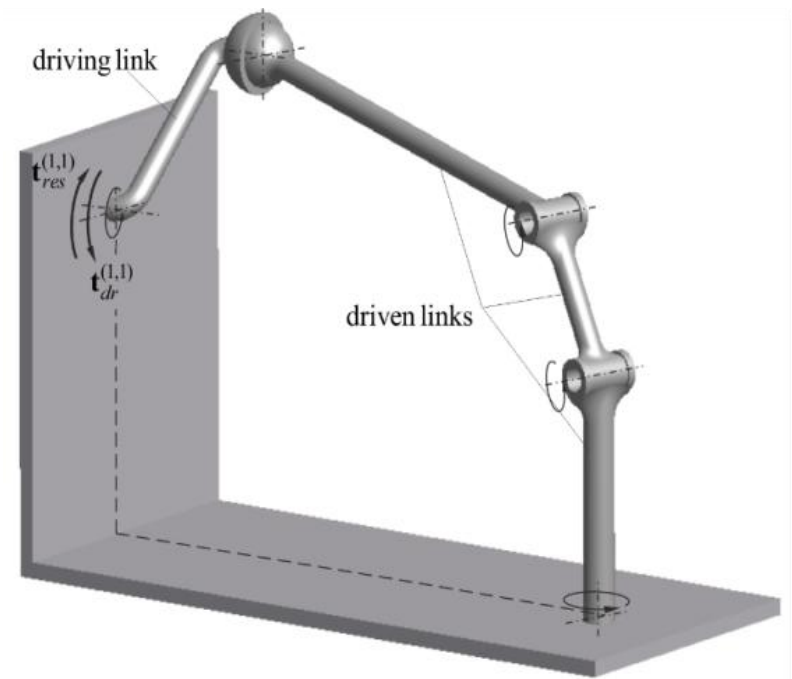

Fig. 1 The analyzed spatial mechanism. 


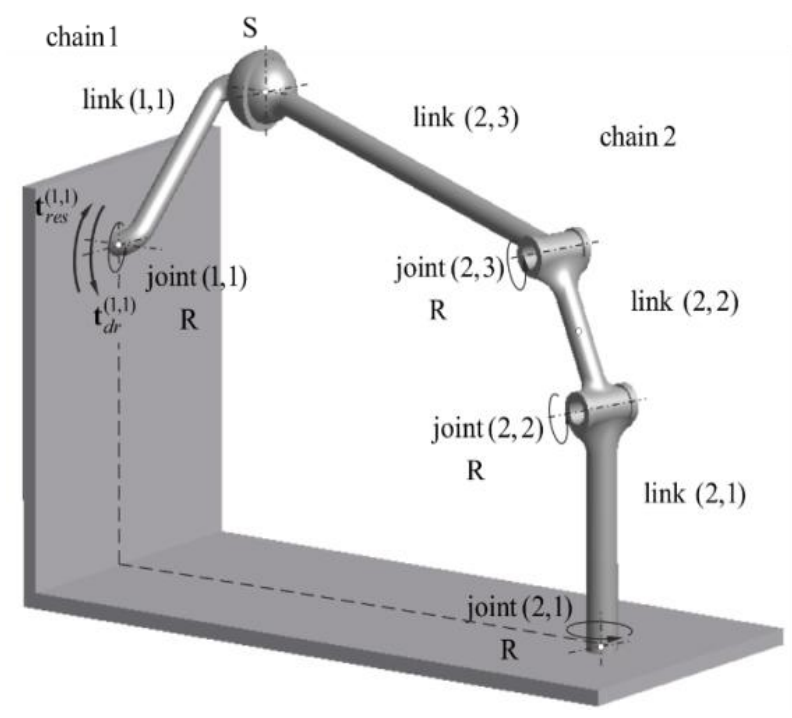

Fig. 2 The system of two open-loop kinematic chains.

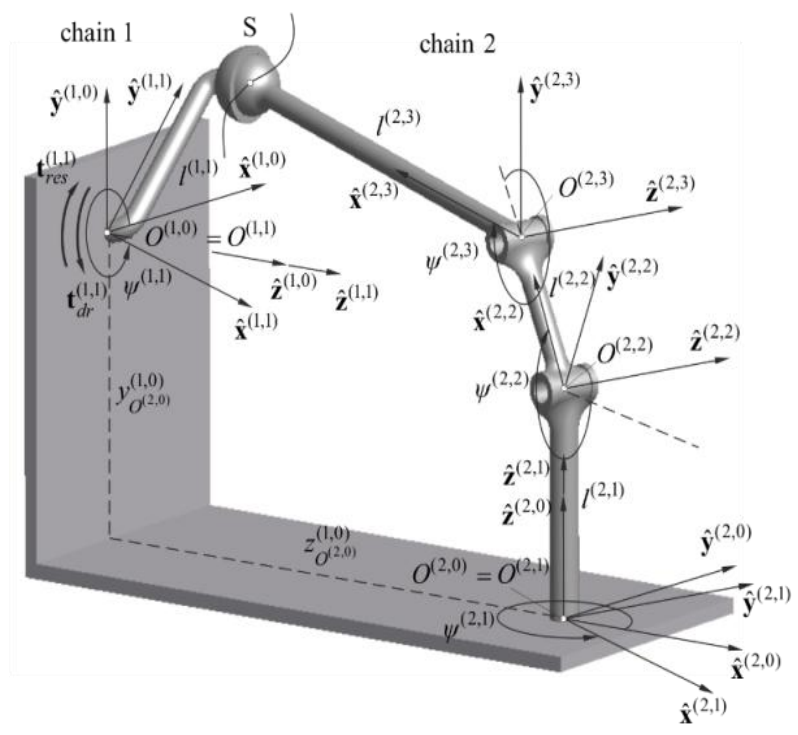

Fig. 3 The assumed coordinate systems.

resulted in obtaining two open-loop kinematic chains joined with the fixed base: 1 - it is formed by the link $(1,1)$, and 2 -it is formed by the links $(2,1),(2,2)$, $(2,3)$. The links in the kinematic chains are interconnected by the rotational joints $\mathrm{R}:(1,1)$-in the chain 1 , and $(2,1),(2,2),(2,3)$ - in the chain 2 .

The local coordinate systems, formed by right-handed oriented versors, were attached-according to the Denavit-Hartenberg notation - to the particular links (including the fixed base). The fixed coordinate system $(1,0)$, related to the chain 1 , is understood as the global reference system, and the system $(2,0)$, related to the chain 2 , is the auxiliary reference system (Fig. 3).

The motion of the link $p$ is described by the joint coordinate $\psi^{(c, p)}$ which is a component of the suitable joint coordinate vector:

for the chain 1 :

$$
\mathbf{q}^{(1,1)}=\left(q_{j}^{(1,1)}\right)_{j=1}=\left[\psi^{(1,1)}\right],
$$

for the chain 2 :

$$
\mathbf{q}^{(2,3)}=\left(q_{j}^{(2,3)}\right)_{j=1,2,3}=\left[\begin{array}{lll}
\psi^{(2,1)} & \psi^{(2,2)} \quad \psi^{(2,3)}
\end{array}\right]^{T} .
$$

The homogeneous transformation matrices from the local systems attached to the links to the global reference system $\{1,2\}$ are determined according to the relationship:

$$
\mathbf{T}^{(c, p)}=\mathbf{T}^{(c, p-1)} \tilde{\mathbf{T}}^{(c, p)}
$$

where: $\mathbf{T}^{(c, 0)}=\mathbf{I}$,

$$
\begin{aligned}
& \tilde{\mathbf{T}}^{(1,1)}=\left[\begin{array}{cc}
\tilde{\mathbf{R}}^{(1,1)} & \tilde{\mathbf{r}}_{O^{(1,1)}}^{(1,0)} \\
\mathbf{0} & 1
\end{array}\right]=\left[\begin{array}{cccc}
\mathrm{c} \psi^{(1,1)} & -\mathrm{s} \psi^{(1,1)} & 0 & 0 \\
\mathrm{~s} \psi^{(1,1)} & \mathrm{c} \psi^{(1,1)} & 0 & 0 \\
0 & 0 & 1 & 0 \\
0 & 0 & 0 & 1
\end{array}\right], \\
& \tilde{\mathbf{T}}^{(2,1)}=\left[\begin{array}{cc}
\tilde{\mathbf{R}}^{(2,0)} & \tilde{\mathbf{r}}_{O^{(2,0)}}^{(1,0)} \\
\mathbf{0} & 1
\end{array}\right]\left[\begin{array}{cc}
\tilde{\mathbf{R}}^{(2,1)} & \tilde{\mathbf{r}}_{O^{(2,1)}}^{(2,0)} \\
\mathbf{0} & 1
\end{array}\right]=
\end{aligned}
$$

$$
\begin{aligned}
& =\left[\begin{array}{cccc}
1 & 0 & 0 & 0 \\
0 & 1 & 0 & -y_{O^{(2,0)}}^{(1,0)} \\
0 & 0 & 1 & z_{O^{(2,0)}}^{(1,0)} \\
0 & 0 & 0 & 1
\end{array}\right]\left[\begin{array}{cccc}
\mathrm{s} \psi^{(2,1)} & \mathrm{c} \psi^{(2,1)} & 0 & 0 \\
0 & 0 & 1 & 0 \\
\mathrm{c} \psi^{(2,1)} & -\mathrm{s} \psi^{(2,1)} & 0 & 0 \\
0 & 0 & 0 & 1
\end{array}\right], \\
\tilde{\mathbf{T}}^{(2,2)} & =\left[\begin{array}{cc}
\tilde{\mathbf{R}}^{(2,2)} & \tilde{\mathbf{r}}_{O^{(2,2)}}^{(2,1)} \\
\mathbf{0} & 1
\end{array}\right]= \\
& =\left[\begin{array}{cccc}
\mathrm{c} \psi^{(2,2)} & -\mathrm{s} \psi^{(2,2)} & 0 & 0 \\
0 & 0 & 1 & 0 \\
-\mathrm{s} \psi^{(2,2)} & -\mathrm{c} \psi^{(2,2)} & 0 & l^{(2,1)} \\
0 & 0 & 0 & 1
\end{array}\right],
\end{aligned}
$$




$$
\begin{aligned}
\tilde{\mathbf{T}}^{(2,3)} & =\left[\begin{array}{cc}
\tilde{\mathbf{R}}^{(2,3)} & \tilde{\mathbf{r}}_{O^{(2,3)}}^{(2,2)} \\
\mathbf{0} & 1
\end{array}\right]= \\
& =\left[\begin{array}{cccc}
\mathrm{c} \psi^{(2,3)} & -\mathrm{s} \psi^{(2,3)} & 0 & l^{(2,2)} \\
\mathrm{s} \psi^{(2,3)} & \mathrm{c} \psi^{(2,3)} & 0 & 0 \\
0 & 0 & 1 & 0 \\
0 & 0 & 0 & 1
\end{array}\right],
\end{aligned}
$$

$\mathbf{s} \psi^{(\alpha, \beta)}=\sin \psi^{(\alpha, \beta)}, \mathbf{c} \psi^{(\alpha, \beta)}=\cos \psi^{(\alpha, \beta)}$.

The reaction forces $\mathbf{f}_{S, x}, \mathbf{f}_{S, y}, \mathbf{f}_{S, z}$ and $-\mathbf{f}_{S, x}$, $-\mathbf{f}_{S, y}, \quad-\mathbf{f}_{S, z}$, acting on the chain 1 and 2 respectively, in accordance with the versors' directions of the global reference system $\{1,0\}$ (Fig. 4), are applied in the cutting place of the mechanism, and precisely in the symmetry centre $S$ of the spherical joint $\mathrm{S}$.

\section{A Synthesis of the Equations of Motion and an Algorithm of Their Solution}

The equations of motion of both open-loop kinematic chains were formulated on the basis of Lagrange equations using the algorithm presented in monograph [14]:

$$
\left[\begin{array}{ccc}
\mathbf{A}^{(1,1)} & \mathbf{0} & -\mathbf{D}^{(1,1)} \\
\mathbf{0} & \mathbf{A}^{(2,3)} & \mathbf{D}^{(2,3)} \\
\mathbf{D}^{(1,1)^{T}} & -\mathbf{D}^{(2,3)^{T}} & \mathbf{0}
\end{array}\right]\left[\begin{array}{c}
\ddot{\mathbf{q}}^{(1,1)} \\
\ddot{\mathbf{q}}^{(2,3)} \\
\mathbf{f}_{S}
\end{array}\right]=\left[\begin{array}{l}
\mathbf{e}^{(1,1)}+\mathbf{t}_{d r}^{(1,1)}-\mathbf{t}_{r e s}^{(1,1)}-\mathbf{t}_{f}^{(1,1)} \\
\mathbf{e}^{(2,3)}-\mathbf{t}_{f}^{(2,3)} \\
\mathbf{c}^{(1,2)}
\end{array}\right],
$$

where:

$$
\begin{aligned}
& \mathbf{A}^{\left(c, n_{b}^{(c)}\right)}=\left(\left(\mathbf{A}^{\left(c, n_{b}^{(c)}\right)}\right)_{i, j}\right)_{i, j=1, \ldots, n_{b}^{(c)}},\left(\mathbf{A}^{\left(c, n_{b}^{(c)}\right)}\right)_{i, j}=\sum_{p=\max \{i, j\}}^{n_{b}^{(c)}} \operatorname{tr}\left\{\mathbf{T}_{i}^{(c, p)} \mathbf{H}^{(c, p)} \mathbf{T}_{j}^{(c, p)^{T}}\right\}, \\
& \mathbf{e}^{\left(c, n_{b}^{(c)}\right)}=\left(\left(\mathbf{e}^{\left(c, n_{b}^{(c)}\right)}\right)_{k}\right)_{k=1, \ldots, n_{b}^{(c)}},\left(\mathbf{e}^{\left(c, n_{b}^{(c)}\right)}\right)_{k}=\left(-\mathbf{h}^{\left(c, n_{b}^{(c)}\right)}-\mathbf{g}^{\left(c, n_{b}^{(c)}\right)}\right)_{k}=\sum_{p=k}^{n_{b}^{(c)}}\left[-h_{k}^{(c, p)}-g_{k}^{(c, p)}\right] \\
& h_{k}^{(c, p)}=\sum_{i, j=1}^{p} \operatorname{tr}\left\{\mathbf{T}_{k}^{(c, p)} \mathbf{H}^{(c, p)} \mathbf{T}_{i, j}^{(c, p)^{T}}\right\} \dot{\tilde{q}}_{i}^{(c, p)} \dot{\tilde{q}}_{j}^{(c, p)}, \\
& g_{k}^{(c, p)}=m^{(c, p)} g \boldsymbol{\theta}_{2} \mathbf{T}_{k}^{(c, p)} \tilde{\mathbf{r}}_{C^{(c, p)}}^{(c, p)}, \\
& \boldsymbol{\theta}_{2}=\left[\begin{array}{llll}
0 & 1 & 0 & 0
\end{array}\right] \text {, } \\
& \mathbf{t}_{d r}^{(1,1)}=\left[t_{d r}^{(1,1)}\right], \mathbf{t}_{r e s}^{(1,1)}=\left[t_{r e s}^{(1,1)}\right], \mathbf{t}_{f}^{(1,1)}=\left[t_{f}^{(1,1)}\right], \mathbf{t}_{f}^{(2,3)}=\left[\begin{array}{lll}
t_{f}^{(2,1)} & t_{f}^{(2,2)} & t_{f}^{(2,3)}
\end{array}\right]^{T},
\end{aligned}
$$

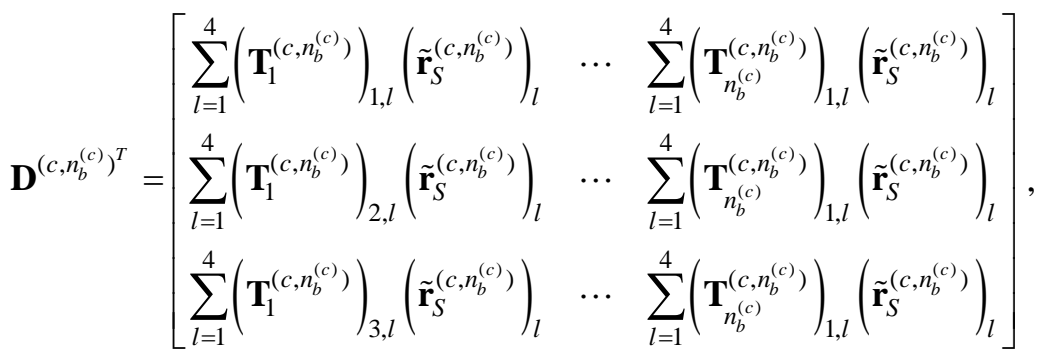




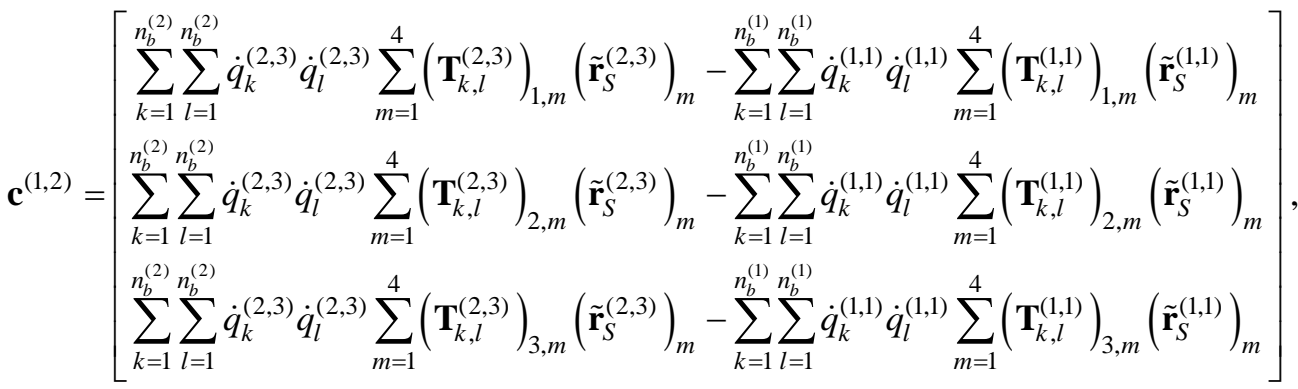

$$
\begin{aligned}
& \mathbf{r}_{S}^{\left(c, n_{b}^{(c)}\right)}=\mathbf{T}^{\left(c, n_{b}^{(c)}\right)} \tilde{\mathbf{r}}_{S}^{\left(c, n_{b}^{(c)}\right)} .
\end{aligned}
$$

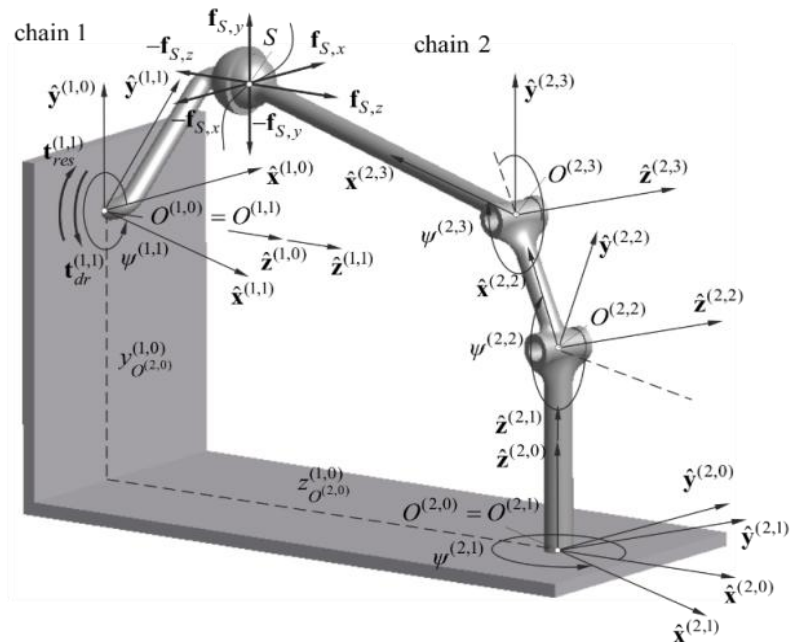

Fig. 4 The reaction forces in the cutting place of the mechanism.

A system of seven ordinary differential equations is obtained which not only contain unknown components of the acceleration vectors $\ddot{\mathbf{q}}^{(1,1)}$ and $\ddot{\mathbf{q}}^{(2,3)}$ but also unknown components of the vector $\mathbf{f}_{S}$.
All these unknown values are determined by using the Gauss elimination method. The system of four differential equations, related to determining the components of the acceleration vectors $\ddot{\mathbf{q}}^{(1,1)}$ and $\ddot{\mathbf{q}}^{(2,3)}$, is solved by the Newmark method with iterative procedure. As mentioned, in order to calculate the values of kinetic friction torques in the particular joints, in each integrating step of the equations of motion, additional calculations are performed using the recursive Newton-Euler algorithm presented in the monograph [13]. In principle, this algorithm is used to calculate joint forces and torques in rotational and prismatic joints of manipulators of robots considered as open-loop kinematic chains.

In the case of an open-loop kinematic chain with links connecting by means of rotational joints, presented in the general form in Fig. 5, the recursive Newton-Euler algorithm has the form [13]:

I. Outward iterations: $p: 0 \rightarrow n_{b}^{(c)}-1$

$$
\begin{aligned}
& \tilde{\boldsymbol{\omega}}^{(c, p+1)}=\tilde{\mathbf{R}}^{(c, p+1)^{T}} \tilde{\boldsymbol{\omega}}^{(c, p)}+\dot{\psi}^{(c, p+1)} \tilde{\hat{\mathbf{Z}}}^{(c, p+1)} \\
& \dot{\tilde{\boldsymbol{\omega}}}^{(c, p+1)}=\widetilde{\mathbf{R}}^{(c, p+1)^{T}} \dot{\tilde{\boldsymbol{\omega}}}^{(c, p)}+\widetilde{\mathbf{R}}^{(c, p+1)^{T}} \dot{\tilde{\boldsymbol{\omega}}}^{(c, p)} \times \dot{\psi}^{(c, p+1)} \tilde{\hat{\mathbf{Z}}}^{(c, p+1)}+\ddot{\psi}^{(c, p+1)} \tilde{\hat{\mathbf{Z}}}^{(c, p+1)} \\
& \dot{\tilde{\mathbf{v}}}_{O^{(c, p+1)}}^{(c, p+1)}=\tilde{\mathbf{R}}^{(c, p+1)^{T}}\left[\dot{\tilde{\boldsymbol{\omega}}}^{(c, p)} \times \tilde{\mathbf{r}}_{O^{(c, p+1)}}^{(c, p)}+\tilde{\boldsymbol{\omega}}^{(c, p)} \times\left(\tilde{\boldsymbol{\omega}}^{(c, p)} \times \tilde{\mathbf{r}}_{O^{(c, p+1)}}^{(c, p)}\right)+\dot{\tilde{\mathbf{v}}}_{O^{(c, p)}}^{(c, p)}\right] \\
& \dot{\tilde{\mathbf{v}}}_{C^{(c, p+1)}}^{(c, p+1)}=\dot{\tilde{\boldsymbol{\omega}}}^{(c, p+1)} \times \tilde{\mathbf{r}}_{C^{(c, p+1)}}^{(c, p+1)}+\tilde{\boldsymbol{\omega}}^{(c, p+1)} \times\left(\tilde{\boldsymbol{\omega}}^{(c, p+1)} \times \tilde{\mathbf{r}}_{C^{(c, p+1)}}^{(c, p+1)}\right)+\dot{\tilde{\mathbf{v}}}_{O^{(c, p+1)}}^{(c, p+1)} \\
& \tilde{\mathbf{f}}_{C^{(c, p+1)}}^{(c, p+1)}=m^{(c, p+1)} \dot{\tilde{\mathbf{v}}}_{C^{(c, p+1)}}^{(c, p+1)} \\
& \tilde{\mathbf{n}}_{C^{(c, p+1)}}^{(c, p+1)}=\mathbf{I}^{(c, p+1)} \dot{\tilde{\boldsymbol{\omega}}}^{(c, p+1)}+\tilde{\boldsymbol{\omega}}^{(c, p+1)} \times \mathbf{I}^{(c, p+1)} \tilde{\boldsymbol{\omega}}^{(c, p+1)}
\end{aligned}
$$

II. Inward iterations: $p: n_{b}^{(c)} \rightarrow 1$ 


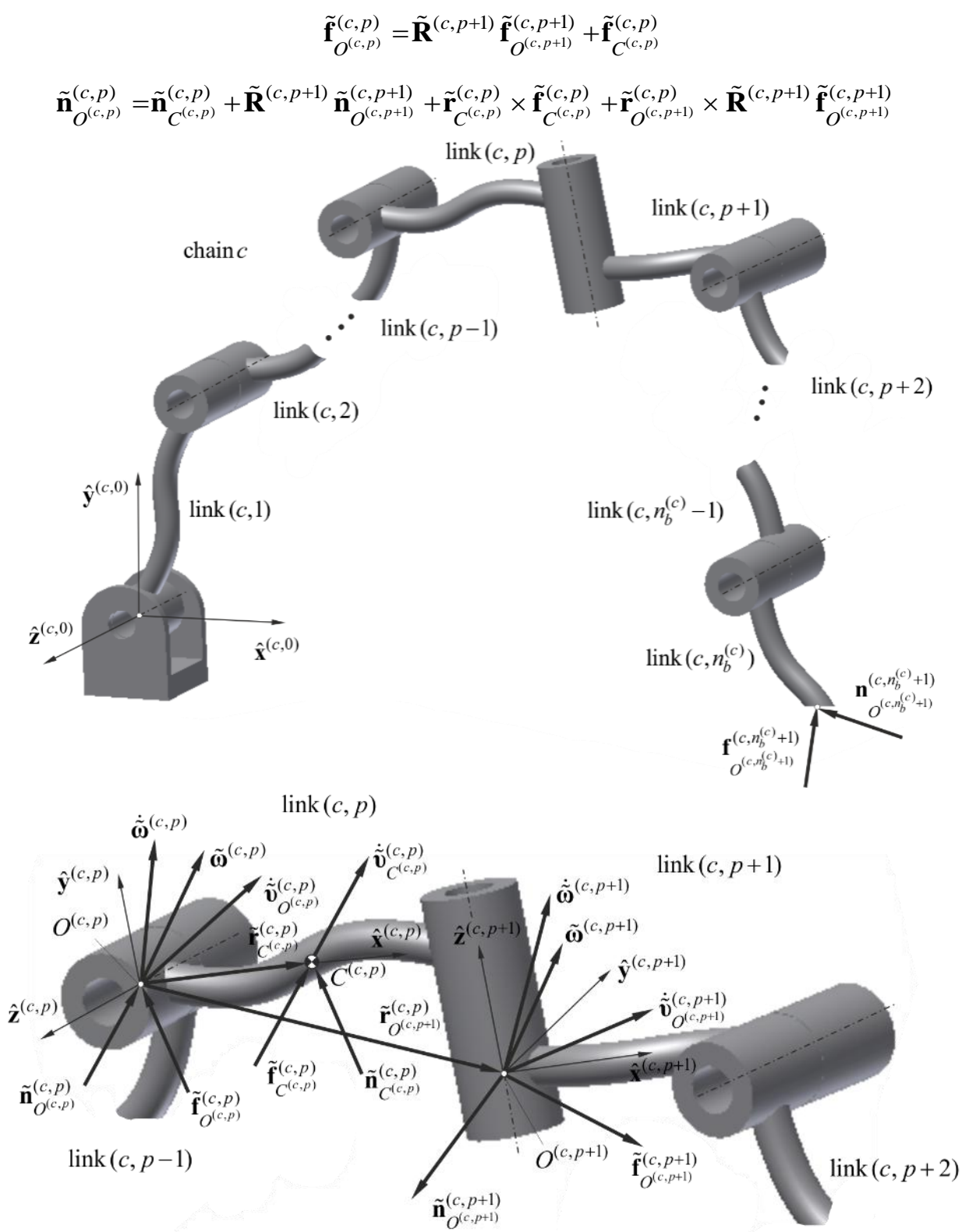

Fig. 5 The general form of an open-loop kinematic chain $c$ with $n_{b}^{(c)}$ links connected by means of rotational joints.

The algorithm presented was used-in the case of the main and auxiliary chain - to determine, in each integrating step of the equations of motion, the joint forces $\tilde{\mathbf{f}}_{O^{(c, p)}}^{(c, p)}$ and torques $\tilde{\mathbf{n}}_{O^{(c, p)}}^{(c, p)}$. Then, the components of these vectors were used to calculate values of reaction forces in the joints and finally the values of kinetic friction torques acting in them. For this purpose, the model of a rotational joint should be prepared. In the literature, models of such joints were presented e.g. in articles [16-18]. For the requirements of the method presented, the model of the rotational joint, as shown in Fig. 6, has been developed. In this model, it is assumed that the journal is part of the link $p-1$, whereas the bearing liner is part of the link $p$. The journal adjoins with the bearing liner by means of friction surfaces - the rotational $A$ and $B$, and the face $C$. In the case of the kinematic chains considered, it can be assumed that the origins of the local coordinate systems attached to the particular links are located in 


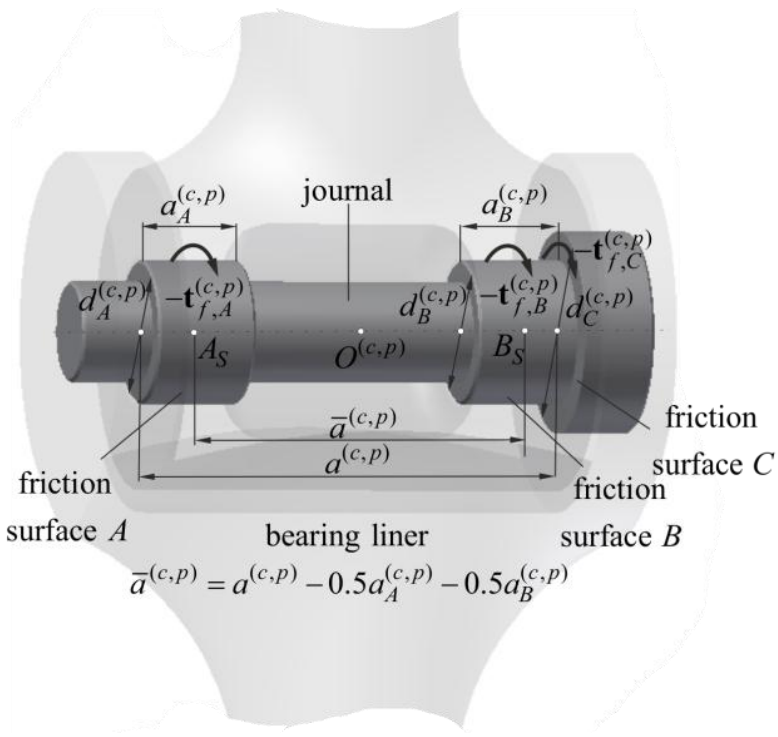

(a)

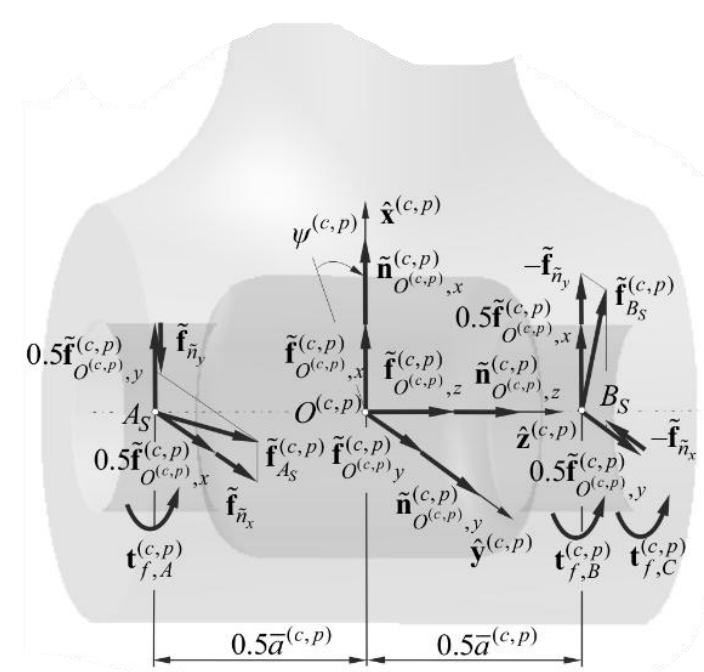

(b)

Fig. 6 The model of the rotational joint connecting link $p$ with link $p-1:$ (a) the pin of link $p-1$; (b) the reaction forces acting on the bearing liner of link $p$.

the axes of joints, in the half-distance $\bar{a}^{(c, p)}$ defining the spacing of the rotational friction surfaces $A$ and $B$.

The value of the kinetic friction torque in the rotational joint was calculated using the following formula:

$$
t_{f}^{(c, p)}=t_{f, A}^{(c, p)}+t_{f, B}^{(c, p)}+t_{f, C}^{(c, p)} .
$$

The values of the kinetic friction torque on the rotational friction surfaces $A$ and $B$ are defined by formulas:

$$
\begin{aligned}
& t_{f, A}^{(c, p)}=\frac{1}{2} \mu_{A}^{(c, p)} \tilde{f}_{A_{S}}^{(c, p)} d_{A}^{(c, p)}, \\
& t_{f, B}^{(c, p)}=\frac{1}{2} \mu_{B}^{(c, p)} \tilde{f}_{B_{S}}^{(c, p)} d_{B}^{(c, p)},
\end{aligned}
$$

whereas on the face friction surface $C$ by means of the formula taken from monographs [19, 20]:

$$
t_{f, C}^{(c, p)}=\frac{1}{3} \mu_{C}^{(c, p)}\left|\tilde{f}_{O^{(c, p)}, z}^{(c, p)}\right| \frac{d_{C}^{(c, p)^{3}}-d_{A}^{(c, p)^{3}}}{d_{C}^{(c, p)^{2}}-d_{A}^{(c, p)^{2}}} .
$$

The values of the reaction forces acting on the rotational friction surfaces $A$ and $B$ are described by formulas:

$$
\tilde{f}_{A_{S}}^{(c, p)}=\sqrt{\left(\frac{1}{2} \tilde{f}_{O^{(c, p)}, x}^{(c, p)}-\tilde{f}_{\tilde{n}_{y}}\right)^{2}+\left(\frac{1}{2} \tilde{f}_{O^{(c, p)}, y}^{(c, p)}+\tilde{f}_{\tilde{n}_{x}}\right)^{2}},
$$

$$
\tilde{f}_{B_{S}}^{(c, p)}=\sqrt{\left(\frac{1}{2} \tilde{f}_{O^{(c, p)}, x}^{(c, p)}+\tilde{f}_{\tilde{n}_{y}}\right)^{2}+\left(\frac{1}{2} \tilde{f}_{O^{(c, p)}, y}^{(c, p)}-\tilde{f}_{\tilde{n}_{x}}\right)^{2}},
$$

where:

$$
\tilde{f}_{\tilde{n}_{x}}=\frac{\tilde{n}_{O^{(c, p)}, x}^{(c, p)}}{\bar{a}^{(c, p)}}, \quad \tilde{f}_{\tilde{n}_{y}}=\frac{\tilde{n}_{O^{(c, p)}, y}^{(c, p)}}{\bar{a}^{(c, p)}} .
$$

In the method presented, only phases of relative motion in the joints of kinematic chains, i.e. phases of kinetic friction, were taken into account. If the rigidity of the drive is sufficiently large, the phases of static friction in the joints (when the values of relative velocities in them decrease to zero) are negligibly short and thus can be omitted. Such a procedure is often used in the case of a dynamic analysis of linkage mechanisms with friction in joints. In order to realize it, one can express the kinetic friction coefficient as a continuous function of relative velocity in a joint (this function in the paper was called kinetic friction characteristic). Generally, in literature, the four main forms of these characteristics are met: the segments-linear characteristic [21] and the three types of non-linear characteristics, i.e. in the form of exponential function [22], hyperbolic tangent [23] and 


$$
\left.\mu_{\alpha}^{(c, p)}\right|_{\alpha \in\{A, B, C\}}=\left\{\begin{array}{l}
\mu_{\alpha, 0}^{(c, p)}\left(1-e^{\left.-k^{(c, p)} \frac{\left|\dot{\psi}^{(c, p)}\right|}{\dot{\psi}_{k}^{(c, p)}}\right)} \text { for } \dot{\psi}^{(c, p)} \geq 0\right. \\
-\mu_{\alpha, 0}^{(c, p)}\left(1-e^{k^{(c, p)} \frac{\dot{\psi}^{(c, p)} \mid}{\dot{\psi}_{k}^{(c, p)}}}\right) \text { for } \dot{\psi}^{(c, p)}<0 .
\end{array}\right.
$$

cubic function $[24,25]$. In the case of this paper, the kinetic friction characteristic, presented in Fig. 7, was taken into account:

The procedure assumed, including the calculations carried out in the scope of the static and dynamic analysis of both open-loop kinematic chains, is presented in Fig. 8.

\section{Numerical Calculation Results}

The geometrical parameters of the particular links and the journals (bearing liners) of the joints, and also the kinetic friction characteristic are presented in Table 1.

It has been assumed that at the initial moment of the mechanism motion, the symmetry axes of all its links are in the vertical plane $\hat{y}^{(1,0)} \hat{z}^{(1,0)}$ of the global reference system $\{1,0\}$ (Fig. 9).

The assumed time courses of the value of the driving torque $t_{d r}^{(1,1)}$ and the resistance torque $t_{\text {res }}^{(1,1)}$, reduced to the axis of the joint connecting link $(1,1)$ with the fixed base, have been presented in Figs. 10a and $10 \mathrm{~b}$, respectively. These courses are to provide that the angular velocity of the driving link will oscillate around a selected value $\dot{\psi}_{0}^{(1,1)}$. The velocities of the other links will fix themselves around suitable values adequate to the assumed value of the angular velocity of the driving link. In the case considered, the following parameters were taken into account: $t_{d r, 0}^{(1,1)}=10 \mathrm{Nm}, t_{s t}=2 \mathrm{~s}$ and $\dot{\psi}_{0}^{(1,1)}=9 \frac{\mathrm{rad}}{\mathrm{s}}$.

The obtained time courses of the joint coordinates and velocities are shown in Fig. 11, while the time courses of the joint forces and torques are shown in Fig. 12 - both in the case when friction in the joints was taken into account and was omitted. It can be observed that the angular velocity of the driving link oscillates around the assumed value $\dot{\psi}_{0}^{(1,1)}=9 \frac{\mathrm{rad}}{\mathrm{s}}$.

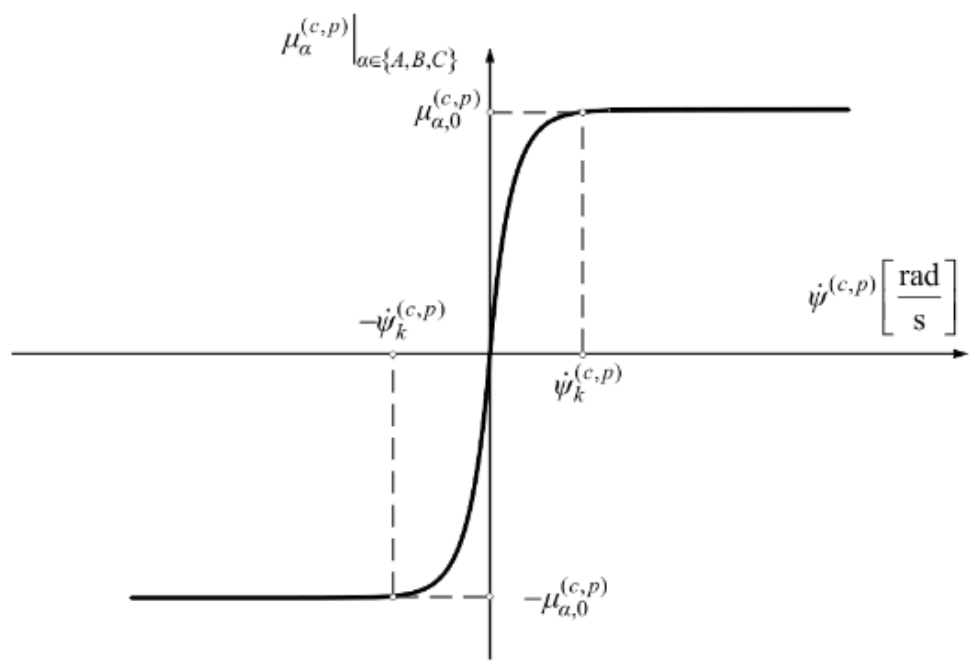

Fig. 7 The general form of kinetic friction characteristic assumed. 


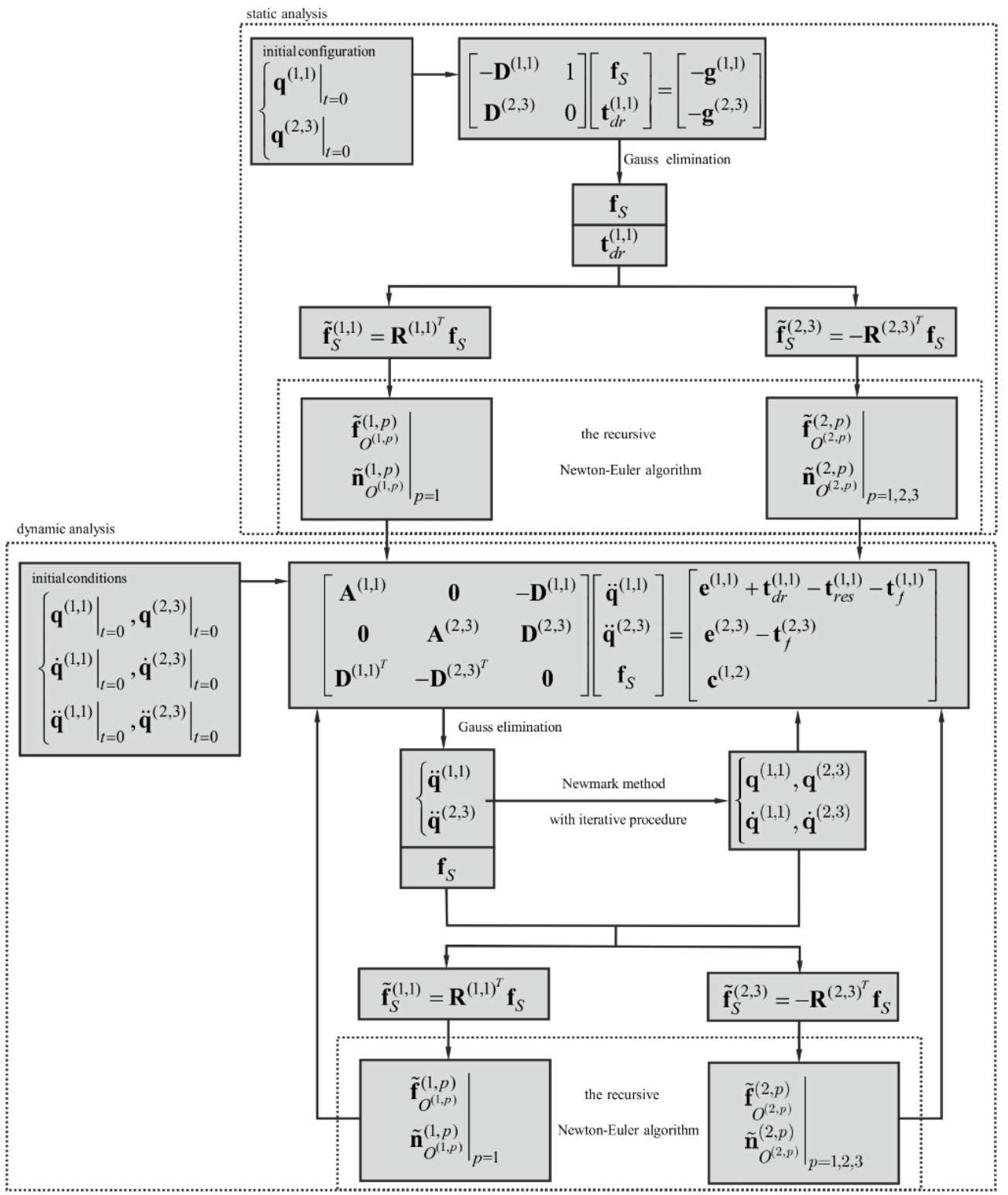

Fig. 8 The algorithms of solving the equations of the static and dynamic analysis of the both kinematic chains.

Table 1 The data for calculations.

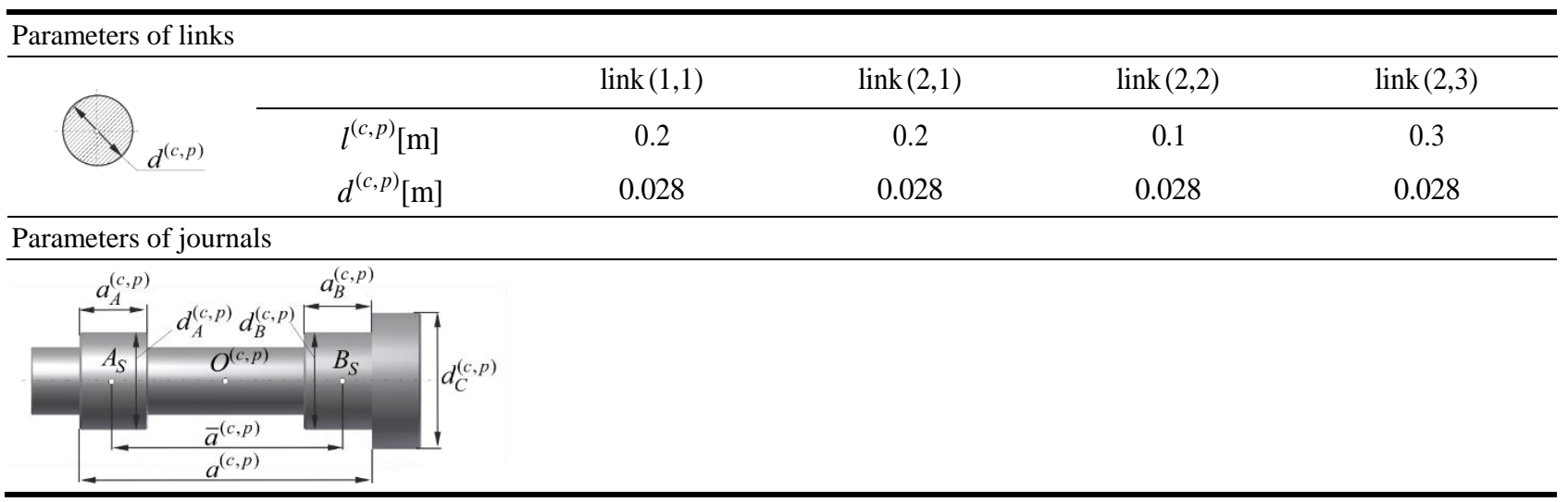


Table 1 to be continued

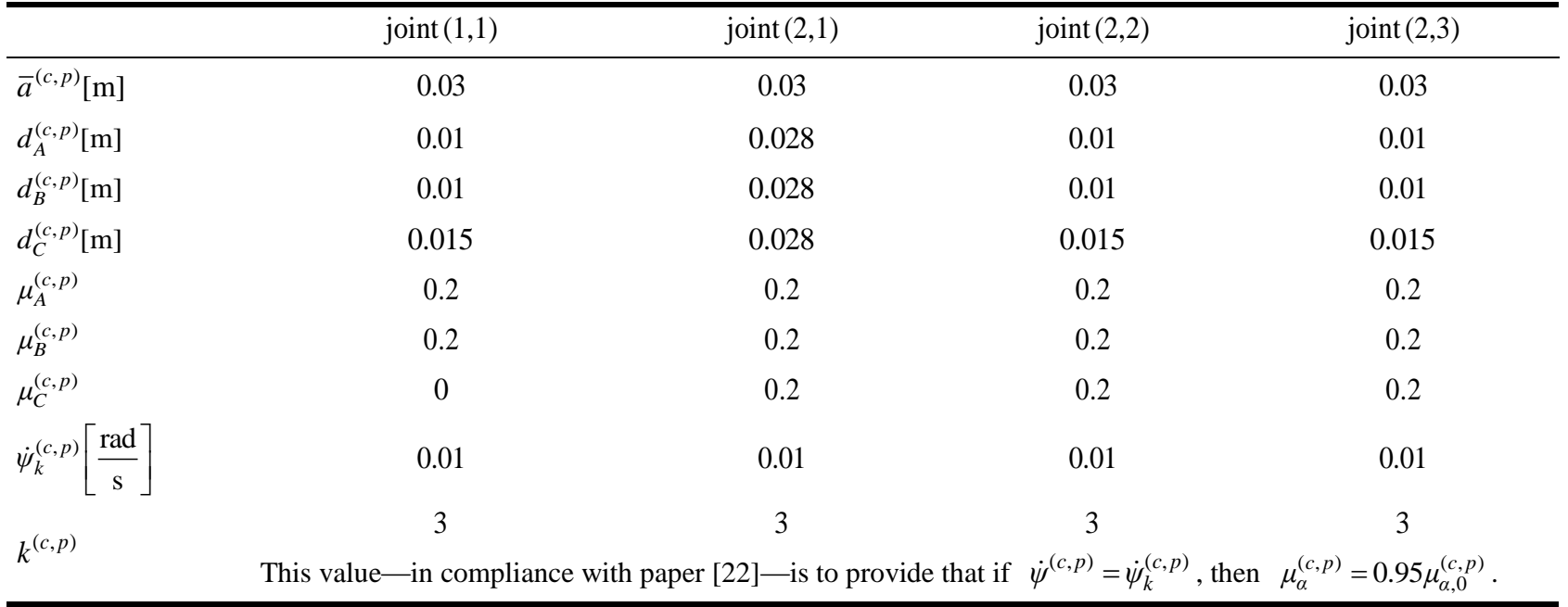

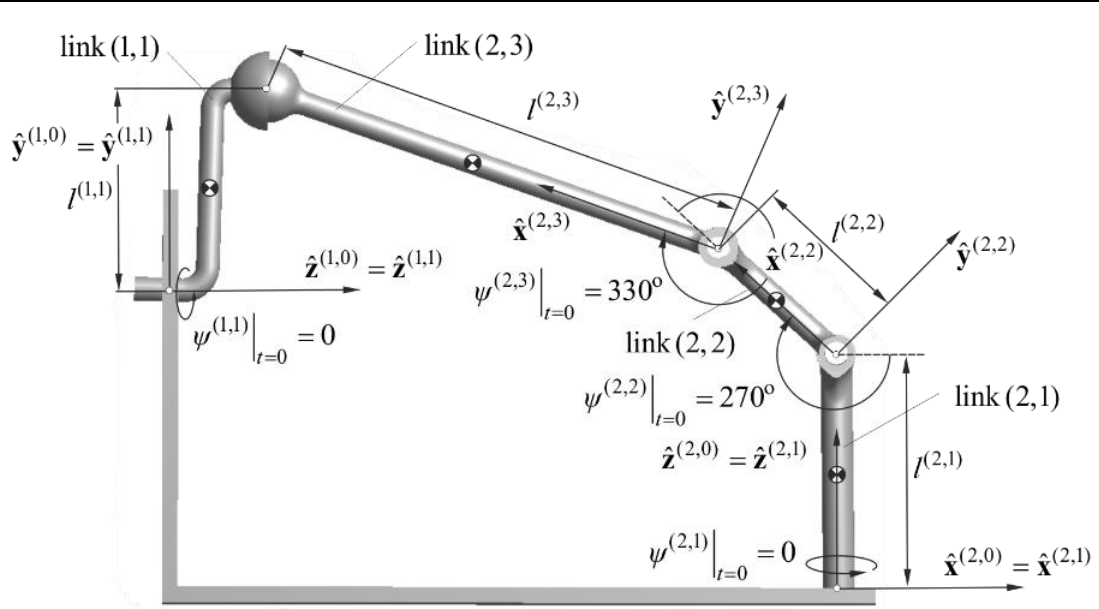

Fig. 9 The initial configuration of the mechanism.

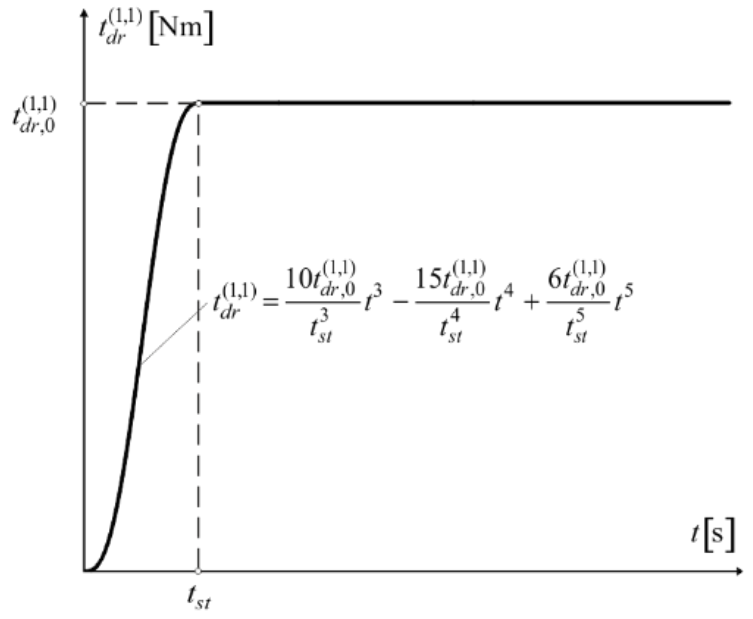

(a)

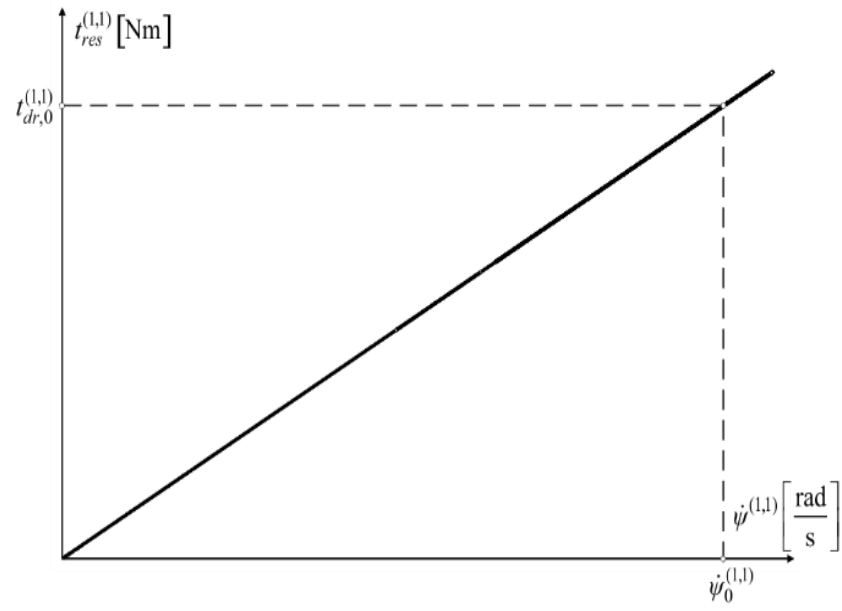

(b)

Fig.10 The course of: (a) the driving torque; (b) the resistance torque. 

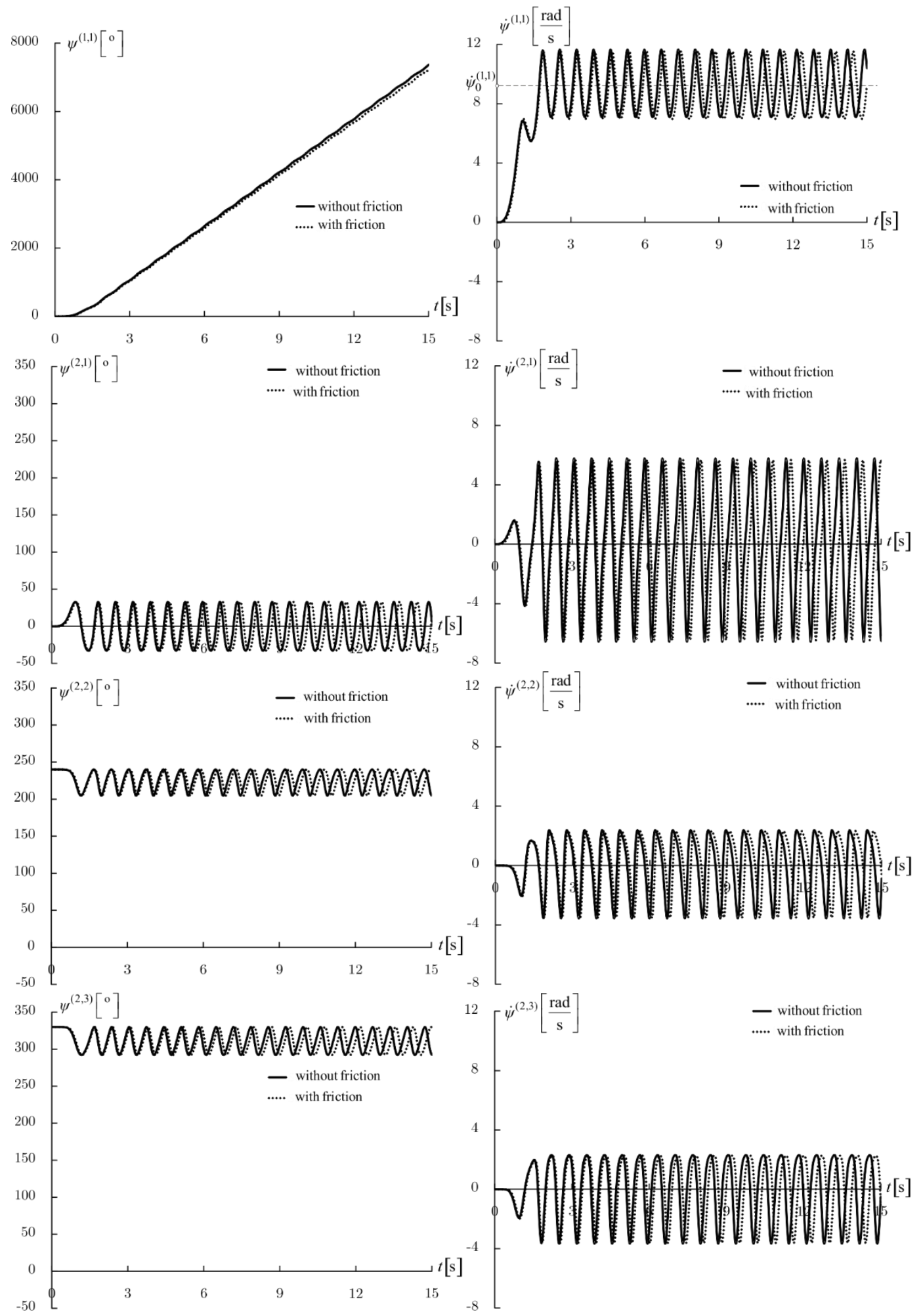

Fig. 11 Time courses of joint coordinates and velocities. 

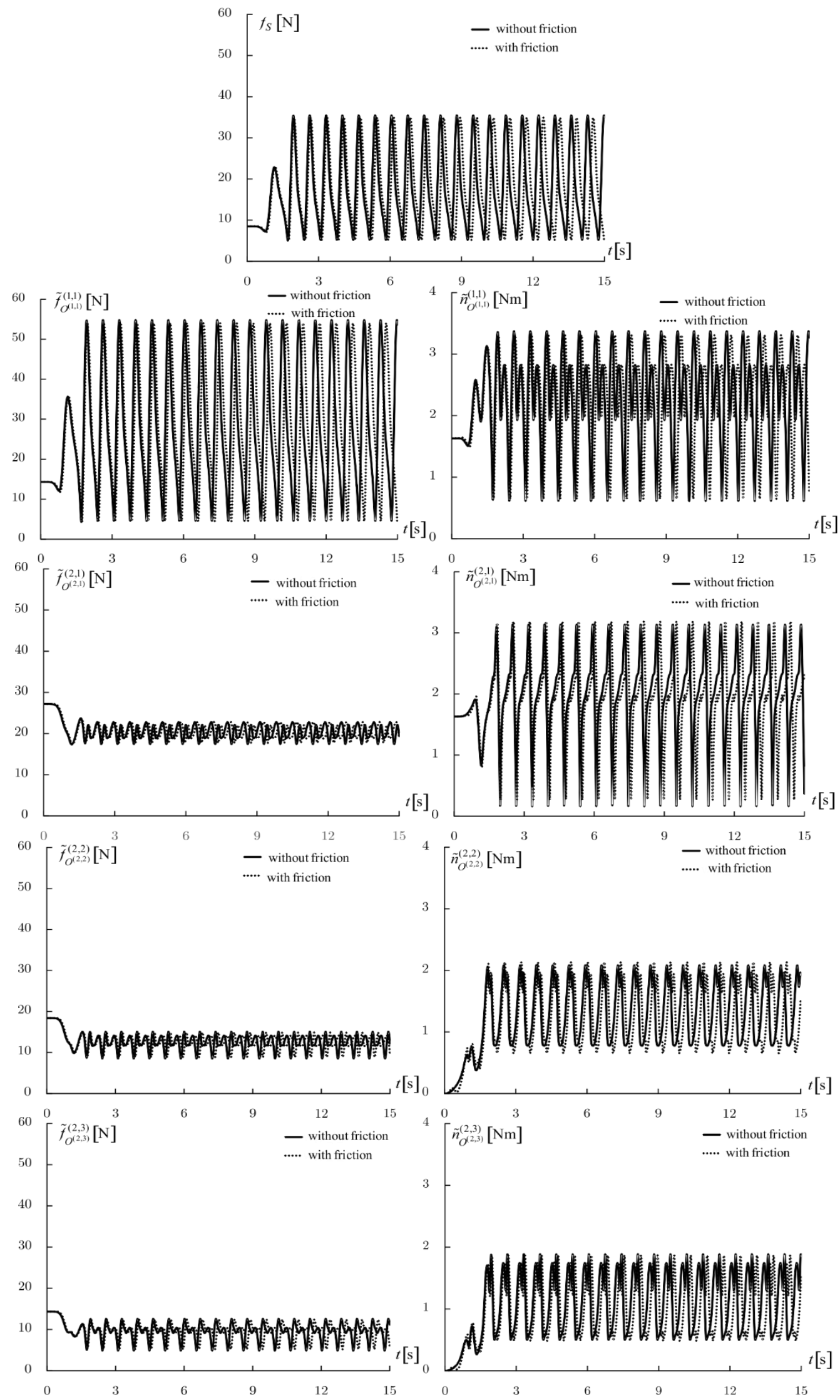

Fig. 12 Time courses of values of joint forces and torques. 
The analysis of the plots presented allows to state that friction significantly slows down the courses (the values of quantities presented in the case of the joints with friction were achieved later than in the case of ideal joints).

\section{Conclusions}

The dynamic analysis of the selected RSRRR spatial linkage mechanism with friction in rotational joints is presented in the article. This mechanism can be qualified to a specific family of the spatial one-DOF linkage mechanisms which contain one spherical joint, a few rotational joints and sometimes one prismatic joint. They do not include neither redundant DOF nor passive constraints in their structure. The proposed method of the analysis assumes a division of the mechanism, in place of the spherical joint, into two open-loop kinematic chains. Owing to this, an effective approach - which is used mainly in the case of the dynamic analysis of the manipulators, in fact being the open-loop kinematic chains - can also be used in the dynamic analysis of such a mechanism. In order to define values of kinetic friction torques, the effective recursive Newton-Euler algorithm, taken from robotics, was used. The numerical experiments proved that only three iterations were required, because the further increasing of accuracy of calculations did not cause perceptible differences in the results obtained. Moreover, these experiments did not claim the need for the constraint stabilization method to increase the accuracy of the numerical calculations. The procedures proposed here have general significance and they could be used to analyze other spatial linkage mechanisms of this family.

\section{References}

[1] Uicker, J. J., Jr. 1965. "On the Dynamics Analysis of Spatial Linkages Using $4 \times 4$ Matrices.” Ph.D. thesis, Illinois.

[2] Denavit, J., and Hartenberg, R. S. 1955. "A Kinematic
Notation for Lower-Pair Mechanisms Based on Matrices." ASME Journal of Applied Mechanics 22: 215-21.

[3] Givens, E. J., and Wolford, J. C. 1968. "Dynamic Characteristics of Spatial Mechanisms." ASME Paper, No.68-Mech-15.

[4] Suh, C. H., and Kang, H. Y. 1989. "A Dynamics Analysis of a Spatial Mechanism with a Passive Degree of Freedom." ASME Journal of Mechanisms, Transmissions, and Automation in Design 111 (2): 238-42.

[5] Garcia de Jalón, J., Unda, J., Avello, A., and Jiménez, J. M. 1987. "Dynamics Analysis of Three-Dimensional Mechanisms in 'Natural' Coordinates." ASME Journal of Mechanisms, Transmissions, and Automation in Design 109 (4): 460-5.

[6] Vilallonga, G., Unda, J., and Garcia de Jalón, J. 1984. "Numerical Kinematic Analysis of Three-Dimensional Mechanisms Using a 'Natural' System of Lagrangian Coordinates." ASME Paper No. 84-DET-199.

[7] Hiller, M., and Kecskemethy, A. A. 1987. "Computer-Oriented Approach for the Automatic Generation and Solution of the Equations of Motion for Complex Mechanisms." In Proc. of 7th World Congress on The Theory of Machines and Mechanisms, edited by Bautista, E., Garcia- Lomas, J., and Navarro, A. Sevilla.

[8] Malczyk, P., and Frączek, J. 2006. "Dynamics Analysis of Complex Closed Loop Kinematic Chains.” In Progress in Robotics. Control, Perceptions, Communication, Communication and Telecommunication Publishers, edited by Tchoń, K. Warsaw, in Polish.

[9] Haug, E. J. 1989. Computer Aided Kinematics and Dynamics of Mechanical Systems: Basic Methods. Allyn and Bacon.

[10] Bae, D. S., and Haug, E. J. 1987. "A Recursive Formulation for Constrained Mechanical System Dynamics: Part I. Open Loop Systems." Mechanics of Structures and Machines 15: 359-82.

[11] Bae, D. S., and Haug, E. J. 1987-1988. "A Recursive Formulation for Constrained Mechanical System Dynamics: Part II. Closed Loop Systems.” Mechanics of Structures and Machines 15: 481-506.

[12] Bae, D. S., Kuhl, J. G., and Haug, E. J. 1988. “A Recursive Formulation for Constrained Mechanical System Dynamics: Part III. Parallel Processor Implementation." Mechanics of Structures and Machines 16: 249-69.

[13] Craig, J. J. 1986, 1989. Introduction to Robotics. Mechanics and Control. Addison-Wesley Publishing Company, Inc.

[14] Jurevič, E. I. (ed.) 1984. Dynamics of Robot Control. Nauka, Moscow, in Russian. 
[15] Baumgarte, J. 1972. "Stabilization of Constraints and Integrals of Motion in Dynamic Systems." Computer Methods in Applied Mechanics and Engineering 1: $1-16$.

[16] Gutkowski, L. J., and Kinzel, G. L. 1992. "A Coulomb Friction Model for Spherical Joints." ASME Journal of Robotics, Spatial Mechanisms and Mechanical Systems DE-Vol. 45.

[17] Sharan, A. M., and Dhanaraj, C. 1993. "A Task Streamlining Approach for Parallel Processing of the Inverse Dynamic Equations with Friction." Trans. of ASME Journal of Dynamic Systems, Measurement, and Control 115 (3).

[18] Wu, S. C., and Yang, S. M., and Haug, E. J. 1986. "Dynamics of Mechanical Systems with Coulomb Friction, Stiction, Impact and Constraint, Addition-Deletion-II. Planar Systems." Mechanism and Machine Theory 21 (5).
[19] Kolčin, N. I. 1972. Mechanics of Machines, Vol. 2. Mašinostrojenije, Leningrad, in Russian.

[20] Koževnikov, S. N. 1969. Theory of Mechanisms and Machines. Mašinostroenije, Moscow, in Russian.

[21] Bernard, J. E. 1980. The Simulation of Coulomb Friction in Mechanical Systems, Simulation.

[22] Threlfall, D. C. 1978. "The Inclusion of Coulomb Friction in Mechanisms Programs with Particular Reference to DRAM." Mechanism and Machine Theory 13 (4).

[23] Ronney, G. T., and Deravi, P. 1982. "Coulomb Friction in Mechanisms Sliding Joints." Mechanism and Machine Theory 17 (1).

[24] Frączek, J. 2002. "Modelling Spatial Mechanisms by Using Multibody Method." Scientific Papers of Warsaw Technical University, No. 196, Warsaw, in Polish.

[25] Ortiz, J. L., and Sohoni, U. 2005. "Joint Friction Modelling in MSC." ADAMS, MSC Software. 